Bangladesh J. Plant Taxon. 27(1): 185-189, 2020 (June)

(C) 2020 Bangladesh Association of Plant Taxonomists

\title{
ITS GENE BASED MOLECULAR GENOTYPING OF NEPETA SHEILAE HEDGE \& R.A. KING (LAMIACEAE) ENDEMIC TO SAUDI ARABIA
}

\author{
Fahad M.A. Alzeibr ${ }^{1}$, M. Ajmal Ali ${ }^{* 2}$, M. Oliur Rahman ${ }^{3}$, \\ Fahad Al-Hemaid ${ }^{2}$, JoOngKu LeE ${ }^{4}$ and SidanAnd V. Kambhar ${ }^{5}$ \\ Department of Botany and Microbiology, College of Science, King Saud University, \\ Riyadh-11451, Saudi Arabia
}

Key words: Nepeta sheilae Hedge \& R.A. King; Lamiaceae; nrDNA; ITS; Endemic; Saudi Arabia.

The genus Nepeta L. (family Lamiaceae), commonly known as 'catmint' or 'catnip', is represented by c. 300 species (Kaya and Dirmenci, 2008), distributed in Asia, Europe, North Africa and America (Jamzad et al., 2000), morphologically characterized by herbaceous, perennial or annuals, sturdy stem and green to greyish-green cordate leaves (Jamzad et al., 2003). In the flora of Saudi Arabia, the genus Nepeta is represented by two species i.e. N. deflersiana Schweinf. and $N$. sheilae Hedge \& R.A. King. N. sheilae is endemic to Saudi Arabia, mainly distributed in northern Hizaz mountains (Chaudhary, 2000). The morphological characters of $N$. sheilae i.e. woody-based, lamina triangular ovate, inflorescence verticillaster, many-flowered, bracteoles narrowly linear-lanceolate, corolla exerted, curved, nutlets brown, apically verrucose or tuberculate etc. overlap with $N$. deflersiana (Chaudhary, 2000). The morphology of $N$. sheilae (Chaudhary, 2000) resembles with section Oxynepeta, and the section Oxynepeta is consistent in the generic classification of Nepeta proposed by Bentham (1848), Briquet (1896) and Budantsev (1993), which are characterized by herbaceous habit; bracts green, inconspicuous; inflorescence interrupted, verticillaster or lax, pedunculate cymes; middle lobe of the lower lip of corolla concave with dentate margin; pollen bi-reticulate, rarely perforate reticulate; and pollen primary muri well-defined, prominent, while secondary muri inconspicuous (Jamzad et al., 2000). Though the phylogenetic relationships in the genus Nepeta and other related genera of Lamiaceae have previously been inferred using ITS sequences of nrDNA, the taxonomic status of $N$. sheilae is unresolved (Jamzad et al., 2003). The nrDNA ITS sequence is well known plant DNA barcoding gene widely applied to represent evolutionary relationships at lower taxonomic ranks, notably at the intrageneric ones (Ali, 2019); hence, the present study aims to resolve the taxonomic status of $N$. sheilae using molecular genotyping of ITS sequence of nrDNA.

The leaves of $N$. sheilae for sequencing were collected from the herbarium specimen [Voucher information: Jabal Lakus Lauz area, South of Haql NW side, 20.5.1990, I.S. Collenette 13417 (RIY)]. The total genomic DNA was isolated using Qiagen DNeasy Plant Mini Kit (Valencia, CA, USA). The nrDNA ITS sequence was amplified using ITS primer (White et al., 1990), and sequenced using ABI PRISM 3100 DNA Analyzer (Perkin-Elmer, Applied Biosystems). In order to unravel the proximity of $N$. sheilae with the members of Nepetoideae, the

\footnotetext{
*Corresponding author, email: ajmalpdrc@gmail.com, majmalaliksu@gmail.com, alimohammad@ksu.edu.sa ${ }^{1}$ Present Address: Department of Biology, Faculty of Sciences, University of Tabuk, Tabuk 71491, Saudi Arabia. ${ }^{2}$ Department of Botany and Microbiology, College of Science, King Saud University, Riyadh-11451, Saudi Arabia ${ }^{3}$ Department of Botany, University of Dhaka, Dhaka 1000, Bangladesh.

${ }^{4}$ Department of Environment and Forest Resources, Chungnam National University, Daehak-ro, Yuseong-gu, Daejeon, Republic of Korea.

${ }^{5}$ Post Graduate Department of Botany, KLE Society's, Basavaprabhu Kore College, Chikodi-591 201, Belagavi, Karnataka, India.
} 
nrDNA ITS sequence of $N$. sheilae was analyzed together with the highly similar sequence revealed from the BLAST search i.e. $N$. heliotropifolia, morphologically similar to $N$. deflersiana, and the representative from six different genera belongs to the family Lamiaceae, subfamily Nepetoideae, and outgroup Paulownia tomentosa (Thunb.) Steud. (family Paulowniaceae) and Pedicularis groenlandica Retz. (family Orobanchaceae) following previous studies ( $\mathrm{Li}$ et al., 2016) retrieved from the GenBank.

The present study revealed that the length of nrDNA ITS region (ITS1, 5.8S and ITS2) in $N$. sheilae sequenced was found to be 640 nucleotides. The generated sequence of $N$. sheilae was submitted to GenBank (accession number MN907379). Among all the Nepeta taxa included in the study, $N$. sheilae showed the highest sequence similarity of $97.66 \%$ with $N$. heliotropifolia (Table 1) in the BLAST-searched (Altschul et al., 1990).

Table 1. Results of BLAST search of nrDNA ITS sequence of Nepeta sheilae.

\begin{tabular}{lllllll}
\hline $\begin{array}{l}\text { Sl. } \\
\text { No. }\end{array}$ & Taxa & $\begin{array}{l}\text { Max. } \\
\text { Score }\end{array}$ & $\begin{array}{l}\text { Total } \\
\text { Score }\end{array}$ & $\begin{array}{l}\text { Query } \\
\text { cover } \\
(\%)\end{array}$ & $\begin{array}{l}\text { Percent } \\
\text { identity } \\
(\%)\end{array}$ & $\begin{array}{l}\text { GenBank } \\
\text { accession } \\
\text { number }\end{array}$ \\
\hline 1 & Nepeta heliotropifolia Lam. & 1099 & 1099 & 100 & 97.66 & AJ515312.1 \\
2. & Nepeta congesta Fisch. \& C.A. Mey. & 1096 & 1096 & 100 & 97.51 & AJ515161.1 \\
3. & Nepeta scrophularioides Rech.f. & 1077 & 1077 & 100 & 97.04 & AJ515319.1 \\
4. & Nepeta cataria L. & 1077 & 1077 & 100 & 97.04 & AJ515313.1 \\
5. & Nepeta kurdica Hausskn. \& Bornm. & 1074 & 1074 & 100 & 96.88 & AJ515320.1 \\
6. & Nepeta isaurica Boiss. \& Heldr. ex & 1064 & 1064 & 100 & 96.72 & AJ515306.1 \\
& Benth. & 1053 & 1053 & 100 & 96.41 & KF765442.1 \\
7. & Nepeta deflersiana Schweinf. & & & & & \\
\hline
\end{tabular}

The phylogenetic analyses of the aligned dataset [CLUSTALX v.1.81 (Thompson et al., 1997)] using Minimum Evolution method (Rzhetsky and Nei, 1992) in MEGA4 (Tamura et al., 2007) were performed. The positions containing gaps and missing data were eliminated from the aligned dataset. There were a total number of 470 positions in the final dataset, out of which 93 were parsimony informative. Variation in the base pair between the sequence of $N$. sheilae and $N$. deflersiana is shown in Fig. 1. The molecular phylogenetic relationships of $N$. sheilae with its closely related $N$. heliotropifolia and $N$. deflersiana, and other members of the subfamily Nepetoideae are illustrated in Fig. 2.

The base pair differences between the sequence of $N$. sheilae and $N$. deflersiana, and the close proximity of $N$. sheilae with $N$. heliotropifolia (bootstrap 47\%) in the phylogenetic tree revealed harmony with the BLAST search result; thus, we herein recognized $N$. sheilae as a distinct species and is different from morphologically similar N. deflersiana. 


\begin{tabular}{|c|c|c|c|c|c|}
\hline \#. $\left.1 \ldots\right|^{10}$ & $\begin{array}{c}20 \\
\text { TGACCTGCGG }\end{array}$ & $\begin{array}{c}30 \\
\ldots \text { AAAGGATCAT }\end{array}$ & $\begin{array}{l}40 \\
\text { TGTCGAAACC }\end{array}$ & $\begin{array}{c}{ }^{50} \\
\text { TGCAAAGCAG }\end{array}$ & $\begin{array}{c}{ }^{60} \\
\ldots \ldots 1 \ldots \\
\text { ACCGCGAACA }\end{array}$ \\
\hline GTTTCCGTAG & GTGAACCTGC & GGAAGGATCA & TTGTCGAAAC & CTGCAAAGCA & GACCGCGAAC \\
\hline$\star \star \star \star \star \star$ & * & * * & $\star \star \star *$ & ** & * \\
\hline$\ldots \ldots|\ldots|^{70}$ & $\begin{array}{r}8^{80} \\
\ldots \ldots|\ldots|^{1} \\
\text { GCAATTCCCG }\end{array}$ & $\begin{array}{r}{ }^{90} \\
\ldots \ldots 1 \ldots \\
\text { GCCGGCGCGC }\end{array}$ & $\begin{array}{l}100 \\
\ldots \ldots \mid \ldots \ldots \\
\text { TCCCCGTGAC }\end{array}$ & $\begin{array}{l}110 \\
\ldots \ldots \mid \ldots 1^{10} \\
\text { GCGCCGCCCG }\end{array}$ & ${ }_{\text {GGCTAACTAA }}^{120}$ \\
\hline $\begin{array}{c}\text { ACGTAACCAA } \\
* * *\end{array}$ & $\underset{* * * *}{\text { CGCAATTCCC }}$ & GGCCGGCGCG & $\underset{* \star *}{\mathrm{CTCCCCCGGG}}$ & $\begin{array}{c}\text { ACGCGCCGCC } \\
* * * \\
* *\end{array}$ & $\underset{*}{\text { CGGGCTAACT }}$ \\
\hline $\begin{array}{c}130 \\
\ldots \ldots \mid \ldots \ldots \\
\text { CCCCGGCGCG }\end{array}$ & $\begin{array}{c}140 \\
\ldots \text { GAATGCGCCA }\end{array}$ & $\ldots \ldots|\ldots|$ & $\ldots \ldots 1 \ldots \ldots 1$ & 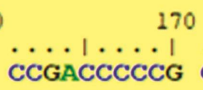 & ${ }^{180}{ }^{181 \ldots \ldots 1}$ \\
\hline $\begin{array}{c}\text { AACCCCGGCG } \\
* *\end{array}$ & $\underset{\text { CGGAATGCGC }}{\text { C. }}$ & $\begin{array}{c}\text { CAAGGAAAAC } \\
* *\end{array}$ & $\underset{*}{\text { CGAACGAAGC }}$ & $\underset{* * *}{\operatorname{GACCGACCCC}}$ & $\underset{* k * k *}{\operatorname{CGCGCCCCGT}}$ \\
\hline $\begin{array}{c}190 \\
\text { GCGGAGCGCG }\end{array}$ & $\begin{array}{c}200 \\
\ldots \text { CGGGGACCG }\end{array}$ & $\ldots$ & TAI . . ${ }^{220}$ & $\begin{array}{c}230 \\
\ldots \ldots \mid \ldots \ldots 1 \\
\text { AACGACTCTC }\end{array}$ & ${ }_{\text {GGCAACGGAT }}^{240}$ \\
\hline $\begin{array}{c}\text { CCGCGGAGCG } \\
* * * * * *\end{array}$ & $\begin{array}{l}\text { CGCGGGGGAC } \\
k_{*} * * *\end{array}$ & $\underset{* *}{\text { CGGCCGTCTA }}$ & TCTATATGTC & $\underset{*}{\text { ATAACGACTC }}$ & TCGGCAACGG \\
\hline $\begin{array}{c}250 \\
\ldots \ldots \mid \ldots \ldots \text { । } \\
\text { ATCTCGGCTC }\end{array}$ & $\begin{array}{c}260 \\
\text { TCGCATCGAT }\end{array}$ & $\ldots \ldots|\ldots|^{270}$ & $\begin{array}{r}280 \\
\ldots \ldots|\ldots|^{2} \\
\text { GCGAAATGCG }\end{array}$ & $\begin{array}{l}{ }^{290} \\
\ldots \ldots|\ldots|^{2} \\
\text { ATACTTGGTG }\end{array}$ & $\begin{array}{l}300 \\
\ldots \ldots \mid \ldots \ldots \\
\text { TGAATTGCAG }\end{array}$ \\
\hline$\underset{* * * *}{\operatorname{ATATCTCGGC}}$ & TCTCGCATCG & $\underset{*}{\text { ATGAAGAACG }}$ & $\underset{*}{\text { TAGCGAAATG }}$ & $\underset{*}{\operatorname{CGATACTTGG}}$ & $\underset{* *}{\text { TGTGAATTGC }}$ \\
\hline$\left.\ldots 1 \ldots 10\right|^{310}$ & $\begin{array}{c}{ }^{320} \\
\ldots \text { ACCATCGAGT }\end{array}$ & CTTTGAACGC & $\begin{array}{c}{ }^{340} \\
\text { AAGTTGCGCC }\end{array}$ & $\begin{array}{r}{ }^{350} \\
\ldots \ldots \mid \ldots \ldots 1 \\
\text { CGAAGCCGTC }\end{array}$ & $\begin{array}{c}360 \\
\ldots \ldots 1 \ldots \ldots \\
\text { AGGCCGAGGG }\end{array}$ \\
\hline AGAATCCCGT & GAACCATCGA & GTCTTTGAAC & GCAAGTTGCG & CCCGAAGCCG & TCAGGCCGAG \\
\hline * $\quad$ * $\quad$ * & * & * * & ** & * & * * \\
\hline $\begin{array}{c}{ }^{370} \\
\ldots \text { CACGTCTGCC }\end{array}$ & $\begin{array}{c}380 \\
\ldots \ldots \mid \ldots \text { । } \\
\text { TGGGCGTCAC }\end{array}$ & $\begin{array}{c}390 \\
\ldots \text { GCATCGCGTC }\end{array}$ & $\ldots \ldots \mid \ldots \ldots$ & $\ldots \ldots 1 \ldots \ldots 1$ & 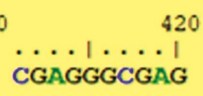 \\
\hline GGCACGTCTG & CCTGGGCGTC & ACGCATCGCG & TCGCCCCCCT & ссестсGстC & GGCGCGGGAG \\
\hline * & * * & ** & $* * * * * *$ & ***** & $\star \star \star * * * *$ \\
\hline$\ldots \ldots|\ldots \ldots|$ & $\ldots$ & $\ldots 1 \ldots{ }^{450}$ & CAIC . ${ }^{460}$ & $\ldots 1 \ldots{ }^{470}$ & $\begin{array}{l}480 \\
\ldots \ldots \text { TGTGATCCGC }\end{array}$ \\
\hline AGGAGGGGCG & GACATTGGCC & CCCCGTGCGC & CCCGGCGCGC & GGCCGGCCCA & AATGTGATCC \\
\hline * ** * & ** & ** **** * & * $\quad * * * * *$ & * $\quad$ * & ** \\
\hline $\begin{array}{c}\quad 490 \\
\text { CGGCGACTCG }\end{array}$ & $\begin{array}{l}500 \\
\ldots \ldots \mid \ldots . \\
\text { TGTCGCGACG }\end{array}$ & AGTGGTGGTT & $\begin{array}{l}520 \\
\ldots \text { GAATTTCTCA }\end{array}$ & $\begin{array}{l}530 \\
\ldots \ldots 1 \ldots \ldots 1 \\
\text { ATCTCGCAGC }\end{array}$ & $\begin{array}{l}540 \\
\ldots \ldots|\ldots| \\
\text { GTCGCGCCCC }\end{array}$ \\
\hline CCCGGCGACT & CGTGTCGCGA & CGAGTGGTGG & TTGAATTCCT & CAATCTCGCA & GCGTCGCGCC \\
\hline * $\quad$ * $\quad$ & $\star \star \quad \star \star \star$ & * * * & * & ** $*$ & * $\quad \star * \star * * *$ \\
\hline $\begin{array}{c}\quad 550 \\
\ldots \ldots|\ldots \ldots| \\
\text { CGTGTCGTCC }\end{array}$ & $\begin{array}{c}\quad 560 \\
\ldots \ldots|\ldots \ldots| \\
\text { GGACGGGCAT }\end{array}$ & $\ldots \ldots 1 \ldots \ldots 1$ & $\begin{array}{r}580 \\
\ldots \ldots|\ldots .| \\
\text { CCCAACGGTG }\end{array}$ & $\begin{array}{l}590 \\
\ldots \ldots \mid \ldots .1 \\
\text { CTGCGCACCA }\end{array}$ & $\begin{array}{l}{ }^{600} \\
\ldots \ldots \mid \ldots .1 \\
\text { TCGACCGCGA }\end{array}$ \\
\hline CCCGTGTCGT & CCGAACGGGC & ATCAACGAAC & GACCCAACGG & TGCCTTGCAC & CATCGACCGC \\
\hline * $\quad * *$ & * & & * & * & ** \\
\hline CC.। . . ${ }^{610}$ & ...। . . ${ }^{620}$ & CCСACTGAGT & TTAAGCAT & & \\
\hline GACCCCAGGT & CAGGCGGGAT & CACCCGCTGA & GTTTAAGCAT & & \\
\hline
\end{tabular}

Fig. 1. The differences in the nucleotide base pairs in the alignment (sequence lane 1: Nepeta deflersiana; sequence lane 2: N. sheilae; lane 3: Clustal consensus). 


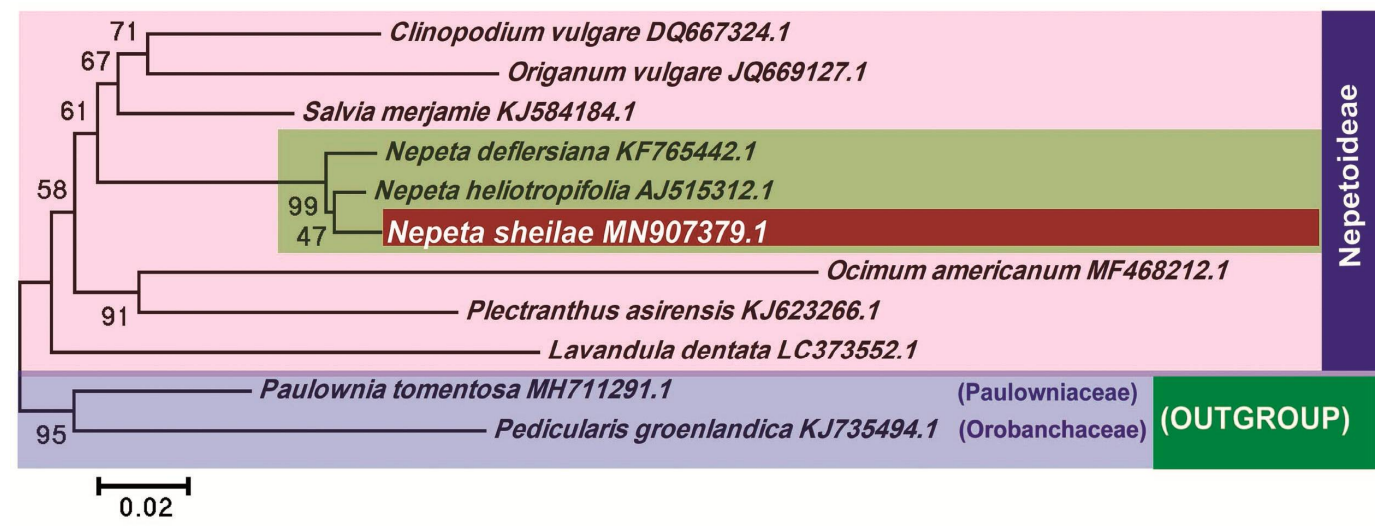

Fig. 2. The phylogenetic tree showing relationships of Nepeta sheilae with its closely related species based on nrDNA ITS sequence data inferred using minimum evolution method. The GenBank accession number shown next to taxon. The bootstrap support in 500 bootstrap replicates shown on the branch.

\section{Acknowledgement}

The authors would like to extend their sincere appreciation to the Deanship of Scientific Research at King Saud University for the funding of this research through the Research Group Project No. RG-1439-84.

\section{References}

Ali, M.A. 2019. Molecular authentication of Anthemis deserti Boiss. (Asteraceae) based on ITS2 region of nrDNA gene sequence, Saudi J. Biol. Sci. 26: 155-159.

Altschul, S.F., Gish, W., Miller, W., Myers, E.W. and Lipman, D.J. 1990. Basic local alignment search tool. J. Mol. Biol. 215(3): 403-410.

Bentham, G. 1848. Labiatae. In: Candolle, A. (Ed.), Prodromus Systematis Naturalis Regni Vegetabilis, Vol. 12. Treuttel and Wurtz, Paris, pp. 27-603.

Briquet, J. 1896. Nepeta, Labiatae. In: Engler, A. and Prantel, K. (Eds), Die Natürlichen Pflanzenfamilien, Teil 4, Abt. 3a. W. Engelmann, Leipzig, p. 235.

Budantsev, A.L. 1993. A synopsis of the genus Nepeta (Lamiaceae). Bot. Zhurn. 78: 93-107. [In Russian].

Chaudhary, S.A. 2000. Flora of the Kingdom of the Saudi Arabia, Vol. II. Ministry of Agriculture and Water, Riyadh, Saudi Arabia.

Jamzad, Z., Harley, M.M., Ingrouille, M., Simmonds, M.S.J. and Jalili, A. 2000. Pollen exine and nutlet surface morphology of the annual species of Nepeta L. (Lamiaceae) in Iran. In: Harley, M.M., Morton, G.M. and Blackmore, S. (Eds), Pollen and Spores: Morphology and Biology. Royal Botanic Gardens, Kew, pp. 385-397.

Jamzad, Z., Ingrouille, M. and Simmonds M.S.J. 2003. Three new species of Nepeta (Lamiaceae) from Iran. Taxon 52: 93-98.

Kaya, T. and Dirmenci, T. 2008. Nutlet surface micromorphology of the genus Nepeta L. (Lamiaceae) in Turkey. Turk. J. Bot. 32: 103-112.

Li, B., Cantino, P., Olmstead, R. Bramley, G.L.C., Xiang, C.-L., Ma, Z.-H., Tan, Y.-H. and Zhang, D.-X. 2016. A large-scale chloroplast phylogeny of the Lamiaceae sheds new light on its subfamilial classification. Sci. Rep. 6: 34343.

Rzhetsky, A. and Nei, M. 1992. A simple method for estimating and testing minimum evolution trees. Mol. Biol. Evol. 9: 945-967. 
Tamura, K., Dudley, J., Nei, M. and Kumar, S. 2007. MEGA4: Molecular Evolutionary Genetics Analysis (MEGA) software version 4.0. Mol. Biol. Evol. 24: 1596-1599.

Thompson, J.D., Gibson, T.J., Plewniak, F., Jeanmougin, F. and Higgins, G.D. 1997. The Clustal X windows interface: flexible strategies for multiple sequence alignment aided by quality analysis tools. Nucleic Acids Res. 24: 4876-4882.

White, T.J., Bruns, T., Lee, S. and Taylor, J. 1990. Amplification and direct sequencing of fungal ribosomal RNA genes for phylogenetics. In: Innis, M.A., Gelfand, D.H., Sninksky, J.J. and White, T.J. (Eds), PCR protocols: a guide to method and amplifications. Academic Press, San Diego, California, pp. 315-322.

(Manuscript received on 03 February 2020; revised on 12 May 2020) 